\title{
Health beliefs, health anxiety and diagnostic type in food hypersensitivity in adults
}

\section{Melissa Goble, Judith Eberhardt, and Paul van Schaik}

\begin{abstract}
Background: food hypersensitivity is often self-diagnosed, and research into barriers to helpseeking is scarce.

Aims: This study in the United Kingdom sought to establish the relationship between health beliefs, health anxiety and diagnostic type (medically-diagnosed vs. self-diagnosed) in individuals with food hypersensitivity, and qualitatively explored attitudes of self-diagnosed individuals and their barriers to attaining a medical diagnosis. Method: A mixed-methods design involving 107 participants with food hypersensitivity (64 medically-diagnosed and 43 self-diagnosed).

Participants completed an adapted version of the health belief model questionnaire and a health anxiety questionnaire. A subset of six self-diagnosed participants took part in semi-structured interviews.
\end{abstract}

Results: Binary logistic regression showed that health anxiety, perceived susceptibility, and perceived severity were significantly associated with diagnostic type. Qualitative thematic analysis of interviews yielded three themes: control over food, diagnosis and treatment; judgement regarding feeling judged negatively on one's choice of food, and being compared to fad-dieters; and the public's and participants' own lack of perceived severity of food hypersensitivity.

Limitation: The sample was self-selected and therefore not necessarily representative of the population; however, an adult population was examined in an area that has so far largely studied children.

Conclusion: health psychologists should become involved in developing and testing interventions to help those with food hypersensitivity to control and reduce distress. Further researching the 
issues of control, judgement, and perceived severity could help tackle barriers to help-seeking behaviour.

\section{Introduction}

Food hypersensitivity, which includes food allergy and food intolerance (Knibb, 2019), is a growing concern; as many as 35\% of individuals in Europe have reported hypersensitivity, even though only around $3 \%$ of these have been shown to suffer from food allergies (Nwaru et al., 2014; Rona et al., 2007). A food allergy is an adverse health effect arising from a specific immune response that occurs reproducibly on exposure to a given food and can be IgE-mediated or non-lgEmediated. Food intolerance, on the other hand, involves adverse non-immunologic reactions to foods not considered allergens (Boyce et al., 2011). Celiac disease is neither an intolerance nor an allergy; it is an immunologic non-IgE-mediated reaction to certain foods (Boyce et al., 2011), and requires the exclusion of gluten from the diet. Food allergy, food intolerance, and celiac disease are all entirely separate health conditions but tend to be seen as the same underlying health problem (Moore, 2014).

It has become apparent over the past two decades that food intolerances are often selfdiagnosed (Young, Stoneham, Petruckevitch, Barton, \& Rona, 1994; Knibb et al., 1999; Biesiekierski, Newnham, Shepherd, Muir, \& Gibson, 2014; Knibb, 2019). Many sufferers seem to be reluctant to return to their doctor to seek help when they are not diagnosed with an illness (Teufel et al., 2007). However, research into the barriers to help-seeking in relation to food health issues is scarce.

Knibb (2019) explored the role of biopsychosocial factors in food hypersensitivity and found that many individuals misdiagnosed their symptoms, which lead to unnecessary dietary restrictions. Therefore, it is important to implement the correct attempts at diagnosis in order to rule out food allergy (Roesler, Barry, \& Bock, 1994). Yet, sufferers often diagnose themselves, without the help of medical professionals. It could be that people who incorrectly self-diagnose are 
unnecessarily exposing themselves to psychological distress. It is unclear what barriers prevent those who self-diagnose from obtaining a medical diagnosis. Due to limited available treatments of food health issues and the difficulty in diagnosis, health-related anxiety and fear surround this ailment (Gowland, 2002; Jones \& Scurlock, 2006). Anxiety has been implicated as a potential driver of patients' help-seeking behaviour (Hu et al., 2002), but the evidence is not consistent (Talley, Boyce, \& Jones, 1997; 1998).

Although symptoms in food health issues have a strong biological component, psychological factors can exacerbate these symptoms (Zopf, Hahn, Raithel, Baenkler, \& Silbermann, 2009). Yet, research into psychological factors is scarce, in particular with regard to the diagnostic process. The health belief model (Rosenstock, 1974) attempts to predict healthrelated behaviour from four central psychosocial factors (perceived susceptibility, perceived severity, perceived barriers, and perceived benefits), with cues to action acting as prompts or reminders to influence the likelihood of the behaviour occurring. The health belief model has been applied to the prediction of a broad range of health behaviours, including eating behaviour (e.g. Deshpande, Basil, \& Basil, 2009; McMorrow, Ludbrook, Macdiarmid, \& Olajide, 2017; Wright, Arce, Himmelgreen, \& Epps, 2019). A systematic review examined the psychosocial factors driving help-seeking behaviour in irritable bowel syndrome and nonulcer dyspepsia (Koloski, Talley, \& Boyce, 2001) and found that perceived severity was a major factor in individuals seeking help from their doctors. However, the model only partially explained help-seeking behaviour. Other variables of the health belief model were not examined, although these may play an important role in shaping health behaviours with these conditions. Furthermore, the systematic review found that abnormal illness attitudes and beliefs are important factors, which help to distinguish those who seek help from those who do not.

Due to the limited research on psychosocial factors in food hypersensitivity, further research is necessary. Moreover, there is evidence that people feel uncomfortable about returning to their doctor when they are not immediately diagnosed with an illness, with sufferers not trusting 
their doctor with regard to diagnosis and treatment, and not seeing their problem as an actual illness in need of medical attention (Teufel et al., 2007). It seems important to examine the health beliefs of those who self-diagnose with food health issues, as opposed to those who receive a medical diagnosis, as this might inform interventions that could support patients in achieving a medical diagnosis for their illness. Therefore, the health beliefs of sufferers of food hypersensitivity need to be examined more closely, in order to understand the relationship between these beliefs and diagnostic type.

Research has suggested that it is important to consider the potential psychological effects of food-related health problems, as there may be an interaction between physical and psychological factors (Teufel et al., 2007). Therefore, psychological correlates of food hypersensitivity should be taken into account. Studies have mainly focused on children, although food health issues can actually be diagnosed at any age (Crespo \& Rodriguez, 2003). Thus, psychological factors in food health issues, such as anxiety, need to be further examined in adults. A growing trend exists that people choose to adhere to restrictive diets ('fad diets'), yet do not have a food hypersensitivity or symptoms (Reilly, 2016). Therefore, it is important to consider the attitudes towards fad-dieters held by individuals who identify as having a food hypersensitivity.

Health beliefs and health anxiety need to be further assessed, comparing sufferers from medically-diagnosed food hypersensitivity with those with self-diagnosed food hypersensitivity, in light of the finding that individuals with self-diagnosed food intolerances may be more likely to suffer from anxiety, insomnia and severe depression than those without an intolerance (Knibb et al., 1999). High levels of anxiety have been found in individuals with self-reported food hypersensitivity, compared to healthy controls (Lillestøl et al., 2010; Simren et al., 2010; Addolorato et al., 1998). However, it is unclear whether self-diagnosed individuals differ in health anxiety to medically-diagnosed individuals. Those who self-diagnose may be doing so wrongly, thus potentially subjecting themselves to needless distress. Consequently, it should be assessed if self-diagnosis and medical-diagnosis of food hypersensitivity are associated with health beliefs 
and health anxiety. This could help better understand the relationship between these constructs and diagnostic type, as well as to prevent self-misdiagnosis. However, as it is unclear whether health anxiety acts as a deterrent or a driver of patient help-seeking behaviour, a qualitative investigation is beneficial to explore this further in self-diagnosed individuals. Moreover, health beliefs require further investigation, as previous research found that one aspect of the health belief model, perceived severity, was a major factor in whether patients sought a medical diagnosis (Koloski et al., 2001). Therefore, factors from the health belief model should be further evaluated in relation to diagnostic type in food hypersensitivity.

In sum, this study aimed to investigate how various health psychological constructs, such as health beliefs and health anxiety, are associated with type of diagnosis (medical diagnosis or self-diagnosis) of food hypersensitivity, drawing on variables from the health belief model. Furthermore, the study aimed to qualitatively explore the views of people who had self-diagnosed, and explore their health attitudes and behaviours in more depth.

\section{Materials and Methods}

\section{Design}

A mixed-method cross-sectional design was adopted. The quantitative component was used in order to not only gather data for analysing the relationship between diagnosis type, and health beliefs and anxiety, but also to screen for suitable participants for the subsequent qualitative component (Denscombe, 2008). The qualitative component was included to provide more insight into the perceptions and experiences of sufferers of self-diagnosed food hypersensitivity. The qualitative method enabled the collection of rich and meaningful data for analysis, which could distinguish patterns within variables (Sofaer, 1999) and offer potential additional explanations for the discovered patterns.

The quantitative component consisted of a correlational design with one outcome variable, type of diagnosis, with two levels: medical diagnosis or self-diagnosis. There were six predictor 
variables: (1) health anxiety, and five in the realm of health belief: (2) perceived barriers, (3) cues to action, (4) perceived susceptibility, (5) perceived severity, and (6) perceived benefits.

The qualitative aspect used an inductive approach, employing semi-structured interviews. This approach was appropriate because it does not make assumptions, but rather allows findings to emerge from the themes in the data (Thomas, 2006). Furthermore, semi-structured interviews were suitable as they offered structure, but allowed deviating from the order of the questions and exploring answers fully (Barriball \& While, 1994). Moreover, probing in interviews can help to develop a sense of rapport, which can help mitigate the limitations of online interviewing and social desirability (Patton, 1990). Face-to-face interviews were used where possible; however, in some circumstances online interviews were necessary due to a long distance between researcher and participant.

\section{Participants}

According to power analysis for logistic regression (Faul, Erdfelder, Lang, \& Buchner, 2007 ), with power $=0.80, \alpha=0.05, \mathrm{OR}=2$, a sample size of 82 was required. A total of 107 participants were recruited via advertisement on the lead author's institution's research participation scheme and social media, such as Facebook pages. This allowed for a snowballing technique to be adopted To be included in the study, individuals needed to report having a food allergy or a food intolerance, or a different type of adverse reaction. Individuals were also asked to indicate whether they had been medically diagnosed or self-diagnosed with a food allergy or food intolerance. There were six males and 101 females, with an age range of 18 to 63 , and a mean age of $28(S D=8.61)$. All participants were included in the quantitative part of the study. A random sample from those who had self-diagnosed their food hypersensitivity, were asked to participate in the qualitative aspect of the study. One male and five females, with an age range of 23 to 31 , and a mean age of 26 , were invited, and took part in, the semi-structured interviews. This sample size was deemed sufficient, as analysis reached saturation, with no new themes being generated from the data (Francis et al., 2010). 


\section{Measures and materials}

The quantitative part of the study included three questionnaires. The demographic questionnaire measured the type of diagnosis (medically diagnosed or self-diagnosed), age, gender, ethnicity, type of diagnosis, food restriction, and length of time of food restriction.

The second questionnaire was an adapted version of the Health Belief Model Questionnaire (Jones et al., 2014; see Supplementary Material A). The final measurement instrument was the Health Anxiety Questionnaire, consisting of 21 items (Lucock \& Morley, 1996; see Supplementary Material B). For the qualitative component of the study, a semi-structured interview schedule was constructed (see Supplementary Material C).

\section{Procedure}

Ethical approval for the study protocol was obtained from the Research Ethics Committee at the authors' institution.

\section{Online questionnaire}

Participants were sent a link electronically to access the information sheet, consent form, and questionnaires. Once participants had indicated their consent, they completed all three questionnaires. Finally, participants were thanked and informed that they would be sent a debrief sheet via email.

\section{Semi-structured interviews}

A subset of six participants were asked to take part in the qualitative component of the study. They were selected via a random number generator, based on their assigned participant numbers, from those participants who had self-diagnosed. Of these six, four were not known personally by the researchers, and two were known in passing by the lead author. Five participants were interviewed face-to-face; however, one could not travel, therefore an online video chat was used. Upon completion of the consent form, participants were asked a series of questions from the interview schedule. Where necessary, the researcher used prompts and probing questions in order to gain richer data. Finally, interviewees received a debrief sheet. 


\section{Analysis}

Descriptive statistics were produced and binary logistic regression analysis was conducted on the quantitative data, with type of diagnosis as the dependent variable and health anxiety, perceived barriers, cues to action, perceived susceptibility, perceived severity, and perceived benefits as the predictors.

The qualitative component of the study used thematic analysis, adopting Braun and Clarke's (2006; 2019) method. This was deemed most suitable, because, while flexible, it also has clear guidelines, enabling an exhaustive and concise analysis of the data (Braun \& Clarke, 2006). All transcripts were analysed by the lead author. Six stages of analysis were followed: the first stage involved familiarisation with the data. This was achieved by transcribing the interview recordings and reading the transcripts several times. Then features of interest were coded on the transcripts. These codes were collated into potential themes, which were then reviewed, and the transcripts re-read. Subsequently names and definitions of the themes were formed and refined accordingly, and then written up.

Trustworthiness, rigour, and quality were assessed throughout the study. The mixedmethods design acted as an across-methodologic triangulation, with both quantitative and qualitative approaches (Barbour, 1998; Boyd, 2000). This helped decrease any bias that may result from using a single method, by counteracting any limitations of one method with the strengths of the other (Mitchell, 1986). Furthermore, the credibility of the study was increased via member-checking after data analysis (Maanen, 1983; Curtin \& Fossey, 2007). All participants confirmed that the themes reported adequately reflected their statements and the results corresponded with what participants wished to communicate to the researcher.

\section{Results}

\section{Quantitative analysis}

Participants' diagnostic type was coded using medical (1) and self (2), as the dependent variable was discrete. The predictors were measured using average scale scores, with health 
anxiety ranging from 0 (low) to 3 (high) and the health belief predictors ranging from 1 (low) to 5 (high). Descriptives by diagnosis type are presented in Table 1. Medically-diagnosed and selfdiagnosed participants differed substantially on all measures (on the effect size measure $d$, all values $>1.3$ and most $>2.7$ ), with higher scores in medically diagnosed participants. [INSERT TABLE 1 HERE]

Health anxiety, and health beliefs (perceived barriers, cues to action, perceived susceptibility, perceived severity, and perceived benefits) did not suffer from multi-collinearity (VIF $<2.5)$. Therefore, binary logistic regression analysis was performed using the forced-entry method to determine the effects of health anxiety, and health beliefs on the likelihood that participants have a medical or self-diagnosis (Table 2).

\section{[INSERT TABLE 2 HERE]}

The logistic regression model was statistically significant, $\chi^{2}(6)=122.68, p<.0005$. The model explained $92.2 \%$ (Nagelkerke $\mathrm{R}^{2}$ ) of the variance in diagnosis type and correctly classified $93.5 \%$ of cases, compared with $59.8 \%$ correctly classified by the null model (without predictors). The results of the Hosmer-Lemeshow test showed a good fit of the model to the data, $\chi^{2}(8)=$ 3.35, $p=0.911$. Health anxiety, perceived susceptibility and perceived severity were independently statistically significant negative predictors of diagnostic type, supporting the descriptive results (Table 1).

\section{Qualitative analysis}

The six self-diagnosed participants interviewed included five females and one male. Table 3 displays their pseudonyms, age, and type of food intolerance.

[INSERT TABLE 3 HERE]

Analysis of the transcripts generated three key themes: Control over Food, Diagnosis and Treatment; Judgement; and Perceived Severity. All themes linked to barriers to seeking a medical diagnosis and to attitudes surrounding food hypersensitivity. 


\section{Theme 1: Control}

Control was a core theme discussed by all participants. It was frequently talked about in relation to control of food.

'If I'm cooking at home I find it quite easy to stick to my diet, like I can control what I have in the cupboards. Like it isn't motivation that makes me break the diet, it's just when I'm out in public I feel like I have a lack of control.' (Emily)

Participants saw controlling their food as easier in their home environment than in public: 'It's being in restaurants or takeaways and not knowing if they are cooking your food with or without wheat in, 'cause they can always make mistakes, especially when no one takes it very seriously.' (Alison)

This perceived lack of control in public, in regard to their food, was seen as a difficulty. There was a lack of trust in food hospitality workers in the sense of ensuring the restricted diet was adhered to; as Alison stated, one could make 'mistakes'. The lack of trust in regard to a lack of control, appeared to be linked to the illness not being taken 'seriously'. This aspect of control was seen negatively by the participants. However, there were aspects of control that were portrayed positively. Two participants discussed control in relation to their illness in a positive light, stating that they could control their food intake. This was perceived as having a positive effect on their health and lifestyle. Health and control were viewed as 'perks' and as having an impact on their ability to live their life well:

'It's a lot easier to cook yourself rather than, like, go out and order takeaways and things. [...] I feel a bit more healthier 'cause l've got that control on what goes in my body.' (Aria) 'I feel like I have more control over my lifestyle and how well I live my life.' (Alison)

However, control over food was not the only control element they elaborated on. Control of diagnosis and treatment was a frequently discussed topic. 
'I had to do it myself in terms of [...] a restricted diet. And then introduce foods [...] one by one and then see if that made me ill.' (Aria)

'I just stop eating wheat, there's no miracle pill to stop it. Why go there to be told I have IBS, nothing seems to get done and I ain't wasting time and waiting about for a diagnosis when I can feel better by just doing it myself.' (Alison)

As participants had self-diagnosed, they felt that the amount of time a diagnosis from a health-care professional took was a specific barrier. They saw the diagnosis as a process they could take control of themselves, and the doctor's diagnosis was repeatedly referred to as being 'pointless'. This was not just the case for the diagnosis, but also for the treatment. Taking control of the diagnostic process and the treatment was linked to the fact there was no cure or 'miracle pill'.

'I don't need the doctor to make me eat it and then go through all this testing to be told to cut wheat out when I've done that anyway.' (Alison)

Treatment of one's illness was felt to be something health-care professionals could not provide, and thus they were not needed in the diagnostic or the treatment process.

Ultimately, control was interpreted positively in relation to food, health and lifestyle. Yet, it was also interpreted negatively as a lack of control when in public food places, related to a lack of trust in food hospitality workers. Participants also discussed control of diagnosis and treatment, where they frequently talked about taking the control from medical professionals. Seeking medical advice from the doctor was seen as being purposeless to the diagnosis and treatment process, as no cure was offered.

\section{Theme 2: Judgement}

Another theme that was generated from the data involved feeling judged by others. Sometimes this involved food hospitality workers and doctors: 
'I feel like personally that the chef might think that l'm being [...] picky with the food, with their food and what they've made. And it's not that, it's not that at all. So I just feel a bit uncomfortable and a bit judged.' (Aria)

'I think some doctors could maybe be a little bit judgy [sic], cause that's what l've had from waiters and other people so, like, I think food intolerances are kinda looked down upon.' (Emily)

Participants recurrently discussed their perception of being judged as picky eaters. This judgement evoked feelings of discomfort and seemed to be connected to the perception of food choice. Participants perceived this judgement to occur because of a misconception of them making a choice to have a restricted diet, rather than being necessary due to an illness.

'I just feel continually judged for making a change because that's the treatment for my illness, but it's seen as a choice when actually it's for my health.' (Alison)

Choice was frequently mentioned by participants, especially when discussing feelings of being judged. They believed that they were judged as making a lifestyle choice to adhere to a restrictive diet, in their view it was a treatment method for their illness. However, negative judgement was not always just talked about in relation to choice. Some participants felt that they were being seen as a fad-dieter, with intolerances being viewed as a non-serious illness. Furthermore, participants often felt themselves to be 'lumped' together with fad-dieters.

'Just get sick of hearing it, oh well I'm gluten free, cause it's a fad. [...] It brings the seriousness down of your intolerance.' (Caleb)

'It's more down to these people who fad diet, they [...] give people a bit of a preconceived opinion of everyone who cuts food out of their diet regardless of their reasons behind it.' (Emily)

Fad-dieters were viewed as the reason behind prejudices against individuals with food hypersensitivity. Participants frequently placed blame on fad-dieters for negatively affecting 
attitudes. In particular, they believed that food hypersensitivity was trivialised because of these fads. This led to a reluctance to adhere to their restricted diet when eating in public with friends: 'I do eat food I shouldn't when I feel like l'd be messing my friends about. So I suppose it's more to do with peer pressure.' (Moana)

'Sometimes we're restricted in where we can go to eat, which can sometimes feel a bit like burdening my friends. [...] I do tend to avoid going out for food, which is then actually meaning I'm missing out on going out and socialising with my friends. But I find that's better than preventing them from going somewhere they really wanna go just 'cause I can't eat there.' (Emily)

Participants claimed they felt like they were being a burden to their friends, in regard to eating out in public. They feared judgement and some stated this was what led them to not adhere to their restricted diet, instead risking the physical consequences. Some participants stated that the fear of being thought of as a burden compelled them to avoid social situations. This led them to miss out on opportunities to socialise.

To summarise, the theme of judgement depicts the extent to which participants perceived judgement and trivialisation of their illness. This judgement came from the general public, but especially food hospitality workers and doctors. Perceived choice of restricted diet was viewed as a reason for stigmatisation. Participants compared intolerances to allergies, with intolerances being deemed less serious. Furthermore, fad-dieters were blamed for contributing to judgement and stigmatisation. This resulted in some participants fearing being a burden to their friends when eating in public, which led them to not adhere to their restricted diet, and sometimes to avoid social situations altogether.

\section{Theme 3: Perceived Severity}

The third theme concerned perceived severity of participants' food hypersensitivity. 
'Food intolerances will always be compared to allergies. But [food intolerances] will always be stigmatized as the not serious one [sic].' (Alison)

'I think that intolerances are totally looked down upon and they're just, like, seen as not an illness [...] because they don't threaten your life.' (Emily)

Participants compared food intolerances with allergies in relation to stigmatisation. They believed that their illness was less serious and not considered as life-threatening. They also perceived this as a reason for why they were judged and their illness was trivialised:

'The celiac disease and nut allergies and things like that, I think they are very serious and they get a lot of medical attention because of, like, the severity of it but food intolerances, I don't think it [sic] does at all' (Hanna)

Participants acknowledged that allergies were more severe and more life-threatening; accordingly they believed that, as a consequence, allergies received more attention. Furthermore, they saw this to be the reason why intolerances were not covered in the news as frequently. ‘I think it’s more just because, like, I won't die from it, like, I can understand why people with allergies wouldn't chance it. But, like, for me it doesn't really matter so long as I'm near a toilet and have no major events the next day.' (Emily)

Due to the perceived lack of severity participants stated they were more likely to not adhere to their restricted diet. However, they accepted experiencing distressing symptoms as a result, because non-adherence was unlikely to cause death:

‘ instantly start to worry, and I suppose that can't help with my stomach 'cause I get a very nervous tummy. So maybe me worrying makes it worse. Like, maybe if I could control my thoughts and my worrying then maybe my symptoms wouldn't be as bad.' (Emily) 
'I start to think about it a lot, like I become very aware that my stomach is gonna start hurting. So, like, maybe it makes it worse for me. And, like, I just really wish I hadn't ate it at that point.' (Alison)

When not adhering to their restricted diet due to this lack of perceived severity, participants admitted feeling regret. They began to worry about anticipated symptoms, which made their physical symptoms worse. While participants perceived their illness as lacking severity and not being life-threatening, they also did not view it as an incurable illness. They voiced their belief that in time their illness would get better.

'It might even just get better, like, my body might eventually be able to digest gluten, I don't really know. And hopefully I'll be able to stop the worrying with time. So yeah I'm pretty positive about the future.' (Alison)

‘I think, like, because I haven't had a proper diagnosis, like, from the doctor I'm hoping that is just, like, a little [...] stint in my life if that makes sense.' (Aria)

This hopeful outlook was attributed to not having received a medical diagnosis, and believing one's illness to be potentially reversible.

In sum, participants acknowledged that intolerances were less severe and less life-threatening than allergies, and they believed this contributed to their trivialisation and a lack of attention being paid to them by society. They also claimed that consequentially they were less likely to adhere to their restricted diets. However, this lack of adherence led to worrying, which in turn they believed made their physical symptoms worse. Participants' lack of perceived severity even encompassed the view that their illness was not long-term. Rather, they had a hopeful outlook that their illness would get better in the future, and attributed this to not having a medical diagnosis.

\section{Discussion}


The present study investigated the relationship between health anxiety and variables from the health belief model to diagnostic type, in people with an official diagnosis of food hypersensitivity, and people with a self-diagnosis of food hypersensitivity. Additionally, self-diagnosed participants' health attitudes and behaviours were qualitatively explored. Logistic regression analysis showed that health anxiety, perceived susceptibility, and perceived severity were associated with type of diagnosis of food hypersensitivity, with medically-diagnosed individuals scoring on average higher in these variables than self-diagnosed individuals. However, perceived barriers, cues to action, and perceived benefits were not associated with type of diagnosis. Although the groups showed substantial differences in perceived barriers, cues to action, and perceived benefits, these differences were not independently statistically significant. Although multi-collinearity was not a threat (see above), the extreme results of the odds ratios for the significant predictors are likely due to simultaneously testing several predictors. Therefore, the effect size $\mathrm{d}$ of the predictors is preferred for interpretation.

The results support the idea that health anxiety would be associated with diagnostic type. Previous research has shown that health anxiety plays a role in food health issues (Gowland, 2002; Jones \& Scurlock, 2006) and acts as a potential catalyst for patients' help-seeking behaviour (Hu et al., 2002), but the present study appears to be the first to examine the relationship between health anxiety and diagnostic type. The idea that health beliefs would be associated with diagnostic type was partially supported, with two factors of the health belief model (perceived susceptibility and perceived severity) being associated with diagnostic type. Medically diagnosed individuals scored higher in all health belief variables, whether or not they were significant. Perceived severity has been found to be a determinant of patients seeking a medical diagnosis for their food-related health problems (Koloski et al., 2001); however, the current study appears to be the first to investigate the relationship between variables of the health belief model and diagnostic type. 
Health anxiety was found to be significantly associated with diagnostic type, with selfdiagnosed participants exhibiting lower health anxiety scores than those who were medicallydiagnosed. However, it is unclear whether this higher level of anxiety was a result of to the definitive medical-diagnosis or whether health anxiety spurred this seeking of a medical-diagnosis. This finding is consistent with the results from a study where anxiety was positively associated with patients seeking help from their doctor for digestive health problems (Hu et al., 2002). Yet, it contradicts the results of research which found no difference in anxiety between those who consulted a doctor and those who did not (Talley, Boyce, \& Jones, 1997; 1998) as well as findings of other research which showed high levels of anxiety in individuals with self-reported food hypersensitivity, compared to healthy controls (Lillestøl et al., 2010; Simren et al., 2010; Addolorato et al., 1998). It is possible that health anxiety is increased with a diagnosis, as patients have had their suspicions confirmed that they have a medical condition. Alternatively it is conceivable that their symptoms were more severe which prompted them to seek medical diagnosis and this severity of symptoms made them more health anxious. Further research, focusing on anxiety and its relationship to diagnosis type, is necessary to establish the nature of this relationship and the reasons for differences in anxiety levels between the two groups.

Perceived susceptibility was also significantly associated with diagnostic type, with medicallydiagnosed participants scoring higher than self-diagnosed ones. Similar findings emerged in previous research on dental problems, where participants were more likely to seek medical help if they had a higher level of perceived susceptibility to a certain dental ailment (Rosenstock, 2005). The role of perceived susceptibility in help-seeking behaviour thus seems to be similar to that in other health-related problems.

Furthermore, perceived severity was significantly associated with diagnostic type, with medically diagnosed individuals also scored higher in perceived severity than those who had selfdiagnosed. It is plausible that either high perceived severity could be a motivating factor to gaining a medical diagnosis, or that having a medical diagnosis could lead to increased perceived 
severity. The findings of the thematic analysis provide further insight, with perceived severity being a main theme. Self-diagnosed participants acknowledged their illness as being less severe than allergies and other medically diagnosed food-health issues. They claimed this made them less likely to adhere to their restricted diet, which in turn led them to worry. Low perceived severity was linked to participants holding the view that their illness was not a long-term condition. It gave them hope that their illness would get better in the future, and they attributed this to the lack of a medical diagnosis. These findings are similar to those of a systematic review, which found that perceived severity was a major factor in individuals seeking help from their doctor (Koloski et al., 2001). The current study has shown that perceived severity may be a factor in seeking a medical diagnosis, with this variable being a significant variable quantitatively as well as a qualitative theme.

Furthermore, qualitative thematic analysis of interviews exploring participants' attitudes and barriers towards obtaining a medical diagnosis identified three main themes: control, judgment, and perceived severity. These themes provide an insight into the perceptions of those who selfdiagnose their illness and the perceptions of others, and indicate potential barriers to not seeking a medical diagnosis. The findings from the qualitative analysis are also in line with those of the above mentioned systematic review which found that attitudes and beliefs are important to help characterise individuals who seek help compared to those who do not (Koloski et al., 2001). The themes control, judgment, and perceived severity were key aspects of the reasons behind selfdiagnosis and a lack of medical-diagnosis. Control was seen as a positive aspect of food hypersensitivity in relation to health, but a negative aspect when there was a lack of control in public food places. However, participants discussed control in relation to their diagnosis and treatment, where they relinquished control from the medical professional. This is similar to findings relating to reasons why men avoid seeking medical help. A number of studies have found that men attribute not going to the doctor to not wanting to relinquish control (Tudiver \& Talbot, 1999; Mansfield, Addis, \& Mahalik, 2003; Mansfield, Addis, \& Courtenay, 2005). However, the current 
study shows that this is also the case for women, where retaining control may contribute to wanting to self-diagnose and self-treat.

The theme of judgment depicts the extent to which participants perceive judgment, stigmatisation, and trivialisation of their illness, with a particular focus on food hospitality workers and doctors. This supports previous research which suggests that individuals do not feel comfortable seeking help from medical professionals in relation to food hypersensitivity due to feeling judged (Teufel et al., 2007); judgment seems to act as a barrier to seeking a medical diagnosis. Participants frequently described being judged for restricting their diet. Previous research has described how individuals felt their illness was trivialised due to there not being a specific medical treatment for it (Moore, 2014). The results of the present study support this and give weight to the claim that food hypersensitivities are not considered as 'real' illnesses (Moore, 2014). The theme also showed that participants placed blame on fad dieters for this judgment.

The theme of perceived severity contained an important element of worry, whereby participants' physical symptoms were described as worsening because of the anticipation that symptoms would present. This supports previous studies which have indicated that psychosomatic symptoms contribute to food intolerance, which is predominantly governed by psychological constructs (Knibb, et al., 1999). Other research found that in food hypersensitivity, individuals who report experiencing physical symptoms almost always report experiencing psychological issues too, such as anxiety and worry (Zopf, et al., 2009). Yet it is unclear whether the psychological symptoms exacerbate the food intolerance, whether they arise from the intolerance itself, or both. An interesting finding constituted some participants' belief that in time, their food hypersensitivity would get better; they did not wish to seek a medical diagnosis, preferring to selfdiagnose, and this lack of formal diagnosis seemed to provide room for the hope that they would get better. However, simultaneously, not having a medical diagnosis was seen to contribute to others' perception of them being on a 'fad diet' and being judged negatively. Thus, both types of diagnosis were seen to have advantages and disadvantages. 


\section{Limitations}

The logistic regression model displayed exceptional goodness of fit. However, the selfselected sample of participants was not necessarily representative of the population, as it included predominantly women in their twenties recruited from a university population. Nevertheless, the use of mixed methods allowed for the limitations of each to be compensated for by the strengths of the other (Mitchell, 1986; Johnson \& Onwuegbuzie, 2004). The qualitative component also gave further insight into the quantitative findings. Furthermore, the study has addressed a gap in existing research on food hypersensitivity; where mainly children had been studied, the current study examined an adult population. Still, further research with a sample more representative of the general population, and a more even gender balance, would be beneficial.

Although the current research differentiated between medically diagnosed and selfdiagnosed individuals with food hypersensitivity, it was not measured how often the self-diagnosed participants attempted to obtain a diagnosis from their health care professionals. Food hypersensitivity can remain undiagnosed for some years (Knibb \& Semper, 2013); at least some of the self-diagnosed individuals may receive a medical diagnosis in the future. Therefore the findings need to be interpreted with caution. Future investigations should assess number of attempts made and/or the length of time for which individuals have been trying to obtain a medical diagnosis.

The cross-sectional nature of the present study does not allow for causal inferences to be made. Prospective studies, following individuals over time to assess causal connections between health beliefs, health anxiety, and diagnostic type, would be useful. Furthermore, this study did not differentiate between different types of food hypersensitivity, or number of hypersensitivities. Future research should examine whether these affect health beliefs and health anxiety.

While participants' age range in the quantitative component of the study was broad (18 - 63 years), the age range within the qualitative component of the study was much narrower $(23-31$ years). A broader age range, achieved through purposive sampling, may have yielded a broader 
Food Hypersensitivity, Health Beliefs, and Diagnostic Type variety of views. Further qualitative exploration of this area should take this into account when sampling.

\section{Conclusion}

This study has shown that psychological factors may be important in food hypersensitivity, with some sufferers perceiving their physical symptoms to be exacerbated by worry. Interventions to reduce distress and provide those with food hypersensitivity with strategies to control worry, may help reduce the perceived severity of physical symptoms, although further research is needed to confirm this. Patients' quality of life could thus be enhanced. Furthermore, physicians' involvement in the diagnostic process could prevent misdiagnoses, which are more likely to occur in self-

diagnosis. This could reduce unnecessary distress. Moreover, based on our results, additional training for food hospitality workers and doctors could help improve the experiences of those with food hypersensitivity. This would not only help tackle the barriers to seeking a medical diagnosis, but possibly reduce the trivialisation of, and negative judgment of those with, food hypersensitivity.

This research has provided new evidence to highlight barriers to help-seeking behaviour in food hypersensitivity, which, if confirmed by further research, could in future be targeted as part of health psychological interventions. The insights gained from this study regarding the trivialisation and underestimation of the influence of food hypersensitivity on individuals provide valuable opportunities for future research. They point to the need for a change in the way that this type of illness is viewed, not only by health-care professionals but by society as a whole.

Declaration of Interest Statement: The authors declare that they have no conflict of interest. 


\section{References}

Addolorato, G., Marsigli, L., Capristo, E., Caputo, F., Dall'Aglio, C., Baudanza, P., . . Gasbarrini, G. (1998). Anxiety and depression: A common feature of health care seeking patients with irritable bowel syndrome and food allergy. Hepato-Gastroenterology, 45(23), 1559-1564.

Barbour, R. S. (1998). Mixing qualitative methods: quality assurance or qualitative quagmire? Qualitative Health Research, 8(3), 352-361. DOI: 10.1177/104973239800800306

Barriball, K. L., \& While, A. (1994). Collecting Data using a semi-structured interview: a discussion paper. Journal of Advanced Nursing, 19(2), 328-335. DOI:10.1111/j.13652648.1994.tb01088

Biesiekierski, J. R., Newnham, E. D., Shepherd, S. J., Muir, J. G., \& Gibson, P. R. (2014).

Characterization of adults with a self-diagnosis of nonceliac gluten sensitivity. Nutrition in Clinical Practice, 29(4), 504-509. DOI: 10.1177/0884533614529163

Boyce, J. A., MD, Assa'ad, A., MD, Burks, A. W., MD, Jones, S. M., MD, Sampson, H. A., MD, Wood, R. A., MD, . . Schwaninger, J. M., MSc. (2011). Guidelines for the diagnosis and management of food allergy in the United States: Summary of the NIAID-sponsored expert panel report. Nutrition Research, 31(1), 61-75. doi:10.1016/j.nutres.2011.01.001

Boyd, C.O. (2000). Combining qualitative and quantitative approaches. In P.L. Munhall \& C.O. Boyd (Eds.), Nursing research: A qualitative perspective (2nd ed., 454-475). Boston: Jones \& Bartlett.

Braun, V., \& Clarke, V. (2006). Using thematic analysis in psychology. Qualitative Research in Psychology, 3(2), 77-101. DOI: 10.1191/1478088706qp063oa

Braun, V., \& Clarke, V. (2019). Reflecting on reflexive thematic analysis. Qualitative Research in Sport, Exercise and Health, 11(4), 589-597. DOI: 10.1080/2159676X.2019.1628806

Crespo, J. F., \& Rodriguez, J. (2003). Food allergy in adulthood. Allergy, 58(2), 98-113. DOI: 10.1034/j.1398-9995.2003.02170.x 
Curtin, M., \& Fossey, E. (2007). Appraising the trustworthiness of qualitative studies: Guidelines for occupational therapists. Australian Occupational Therapy Journal, 54(2), 88-94. DOI: 10.1111/j.1440-1630.2007.00661.x

Deshpande, S., Basil, M. D., \& Basil, D. Z. (2009). Factors influencing healthy eating habits among college students: An application of the health belief model. Health Marketing Quarterly, 26(2), 145-164. doi:10.1080/07359680802619834

Denscombe, M. (2008). Communities of practice: A research paradigm for the mixed methods approach. Journal of Mixed Methods Research, 2(3), 270-283. DOI:

$10.1177 / 1558689808316807$

Faul, F., Erdfelder, E., Lang, A., \& Buchner, A. (2007). GPower 3: A flexible statistical power analysis program for the social, behavioral, and biomedical sciences. Behavior Research Methods, 39(2), 175-191. doi:10.3758/BF03193146

Francis, J. J., Johnston, M., Robertson, C., Glidewell, L., Entwistle, V., Eccles, M. P., \& Grimshaw, J. M. (2010). What is an adequate sample size? Operationalising data saturation for theorybased interview studies. Psychology and Health, 25(10), 1229-1245. DOI:

$10.1080 / 08870440903194015$

Gowland, M. H. (2002). Food allergen avoidance: risk assessment for life. Proceedings of the Nutrition Society, 61(1), 39-43. DOI: 10.1079/PNS2001128

Hu, W. H. C., Wong, W. M., Lam, C. L. K., Lam, K. F., Hui, W. M., Lai, K. C., Xia, H.X.H., Lam, S.K,. \& Wong, B.C.Y. (2002). Anxiety but not depression determines health care-seeking behaviour in Chinese patients with dyspepsia and irritable bowel syndrome: a populationbased study. Alimentary Pharmacology \& Therapeutics, 16(12), 2081-2088. DOI: 10.1046/j.1365-20-36.2002.01377.x

Johnson, R. B., \& Onwuegbuzie, A. J. (2004). Mixed methods research: A research paradigm whose time has come. Educational Researcher, 33(7), 14-26. DOI: $10.3102 / 0013189 \times 033007014$ 
Food Hypersensitivity, Health Beliefs, and Diagnostic Type

Jones, S. M., \& Scurlock, A. M. (2006). The impact of food allergy: the real "fear factor". Annals of Allergy, Asthma \& Immunology, 96(3), 385-386. DOI: 10.1016/S1081-1206(10)60903-9

Jones, C. J., Smith, H. E., Frew, A. J., Toit, G. D., Mukhopadhyay, S., \& Llewellyn, C. D. (2014). Explaining adherence to self-care behaviours amongst adolescents with food allergy: a comparison of the health belief model and the common sense self-regulation model. British Journal of Health Psychology, 19(1), 65-82. DOI: 10.1111/bjhp.12033

Jones, C. J., Smith, H. E., Frew, A. J., Toit, G. D., Mukhopadhyay, S., \& Llewellyn, C. D. (2014). Explaining adherence to self-care behaviours amongst adolescents with food allergy: $\mathrm{A}$ comparison of the health belief model and the common sense self-regulation model. British Journal of Health Psychology, 19(1), 65-82. doi:10.1111/bjhp.12033

Knibb, R. (2019). Why Do People Misdiagnose Themselves with Food Hypersensitivity? An Exploration of the Role of Biopsychosocial Factors. European Medical Journal, 4(1), 30-37.

Knibb, R. C., Armstrong, A., Booth, D. A., Platts, R. G., Booth, I. W., \& MacDonald, A. (1999). Psychological characteristics of people with perceived food intolerance in a community sample. Journal of Psychosomatic Research, 47(6), 545-554. DOI: 10.1016/S00223999(99)00055-0

Knibb, R. C., \& Semper, H. (2013). Impact of suspected food allergy on emotional distress and family life of parents prior to allergy diagnosis. Pediatric Allergy and Immunology, 24(8), 798-803. doi:10.1111/pai.12176

Koloski, N. A., Talley, N. J., \& Boyce, P. M. (2001). Predictors of health care seeking for irritable bowel syndrome and nonulcer dyspepsia: a critical review of the literature on symptom and psychosocial factors. The American Journal of Gastroenterology, 96(5), 1340-1349. DOI: 10.1111/j.1572-0241.2001.03789.x

LillestøI, K., Berstad, A., Lind, R., Florvaag, E., Arslan Lied, G., M.D., \& Tangen, T. (2010). Anxiety and depression in patients with self-reported food hypersensitivity. General Hospital Psychiatry, 32(1), 42-48. doi:10.1016/j.genhosppsych.2009.08.006 
Lucock, M. P., \& Morley, S. (1996). The health anxiety questionnaire. British Journal of Health Psychology, 1(2), 137-150. DOI: 10.1111/j.2044-8287.1996.tb00498.x

Maanen, J. V. (1983). Qualitative Methodology. Beverly Hills: Sage.

Mansfield, A. K., Addis, M. E., \& Courtenay, W. (2005). Measurement of Men's Help Seeking:

Development and Evaluation of the Barriers to Help Seeking Scale. Psychology of Men \& Masculinity, 6(2), 95-108. DOI: 10.1037/1524-9220.6.2.95

Mansfield, A. K., Addis, M. E., \& Mahalik, J. R. (2003). "Why won't he go to the doctor?": The psychology of men's help seeking. International Journal of Men's Health, 2(2), 93-109. DOI: $10.3149 /$ jmh.0202.93

McMorrow L, Ludbrook A, Macdiarmid Jl, et al. (2017) Perceived barriers towards healthy eating and their association with fruit and vegetable consumption. Journal of Public Health 39(2): 330-338. doi:10.1093/pubmed/fdw038

Mitchell, E. S. (1986). Multiple triangulation: A methodology for nursing science. Advances in Nursing Science, 8(3), 18-26.

Moore, L. R. (2014). "But we're not hypochondriacs": The changing shape of gluten-free dieting and the contested illness experience. Social Science and Medicine, 105, 76-83. DOI: 10.1016/j.socscimed.2014.01.009

Nwaru, B. I., Hickstein, L., Panesar, S. S., Muraro, A., Werfel, T., Cardona, V., . . the EAACI Food Allergy and Anaphylaxis Guidelines Group. (2014). The epidemiology of food allergy in Europe: A systematic review and meta-analysis. Allergy, 69(1), 62-75. doi:10.1111/all.12305

Patton, M. Q. (1990). Qualitative Evaluation and Research Methods (2 ${ }^{\text {nd }}$ ed.). Newbury Park, CA: SAGE Publications, Inc.

Reilly, N. R. (2016). The gluten-free diet: recognizing fact, fiction, and fad. The Journal of Pediatrics, 175, 206-210. DOI: 10.1016/j.jpeds.2016.04.014 
Roesler, T. A., Barry, P. C., \& Bock, S. A. (1994). Factitious food allergy and failure to thrive. Archives of Pediatrics \& Adolescent Medicine, 148(11), 1150-1155. DOI: 10.1001/archpedi.1994.02170110036006

Rona, R. J., Keil, T., Summers, C., Gislason, D., Zuidmeer, L., Sodergren, E., . . Madsen, C. (2007). The prevalence of food allergy: A meta-analysis. Journal of Allergy and Clinical Immunology, the, 120(3), 638-646. doi:10.1016/j.jaci.2007.05.026

Rosenstock, I. M. (1974). Historical origins of the health belief model. Health Education \& Behavior, 2(4), 328-335. DOI:10.1177/109019817400200403

Rosenstock, I. M. (2005). Why people use health services. The Milbank Quarterly, 83(4), online only. DOI: 10.1111/j.1468-0009.2005.00425.x

Simrén, M., Månsson, A., Langkilde, A. M., Svedlund, J., Abrahamsson, H., Bengtsson, U., \& Björnsson, E. S. (2001). Food-related gastrointestinal symptoms in the irritable bowel syndrome. Digestion, 63(2), 108-115. doi:10.1159/000051878

Sofaer, S. (1999). Qualitative methods: what are they and why use them? Health Services Research, 34(5), 1101-1118.

Talley, N. J., Boyce, P. M., \& Jones, M. (1997). Predictors of health care seeking for irritable bowel syndrome: a population based study. Gut, 41(3), 394-398. DOI: 10.1136/gut.41.3.394

Talley, N. J., Boyce, P., \& Jones, M. (1998). Identification of distinct upper and lower gastrointestinal symptom groupings in an urban population. Gut, 42(5), 690-695. DOI: 10.1136/gut.42.5.690

Teufel, M., Biedermann, T., Rapps, N., Hausteiner, C., Henningsen, P., Enck, P., \& Zipfel, S. (2007). Psychological burden of food allergy. World Journal of Gastroenterology: WJG, 13(25), 3456-3465. DOI: 10.3748/wjg.v13.i25.3456

Thomas, D. R. (2006). A general inductive approach for analyzing qualitative evaluation data. American Journal of Evaluation, 27(2), 237-246. DOI: 10.1177/1098214005283748 
Food Hypersensitivity, Health Beliefs, and Diagnostic Type

Tudiver, F., \& Talbot, Y. (1999). Why don't men seek help? Family physicians' perspectives on help- seeking behavior in men. Journal of Family Practice, 48(1), 47-52.

Wright, L., Arce, K. S., Himmelgreen, D., \& Epps, J. B. (2019). Farm2Fork: Use of the health belief model to increase fresh fruit and vegetable intake among food pantry participants. Journal of Hunger \& Environmental Nutrition, 14(1-2), 252-261.

doi:10.1080/19320248.2018.1538920

Young, E., Stoneham, M. D., Petruckevitch, A., Barton, J., \& Rona, R. (1994). A population study of food intolerance. The Lancet, 343(8906), 1127-1130. doi:10.1016/S01406736(94)90234-8

Zopf, Y., Hahn, E. G., Raithel, M., Baenkler, H. W., \& Silbermann, A. (2009). The differential diagnosis of food intolerance. Deutsches Ärzteblatt International, 106(21), 359-370. DOI: 10.3238/arztebl.2009.0359 


\section{Table 1}

Mean and standard deviation of health anxiety and health belief scores for both medical and self-diagnosis.

\section{Diagnosis type}

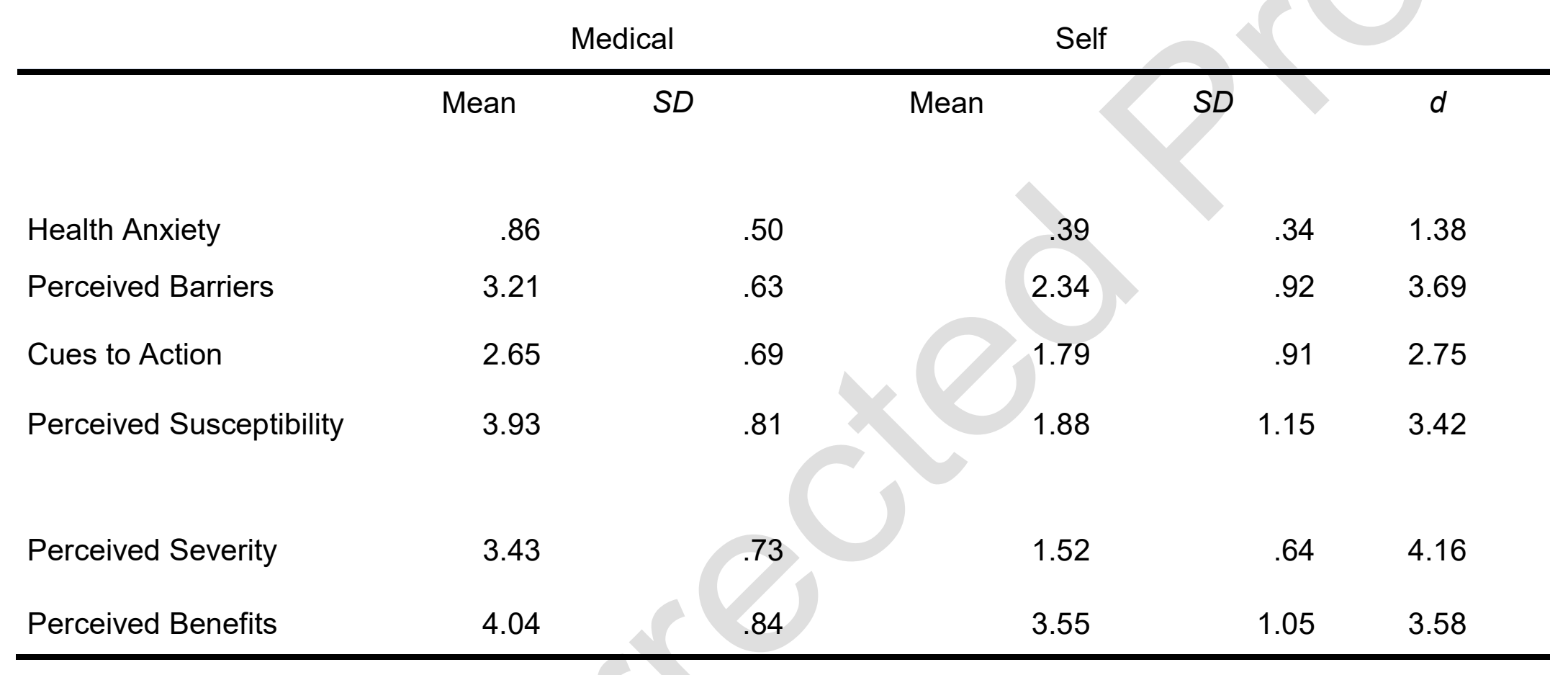


Table 2

Logistic regression summary table for predictors.

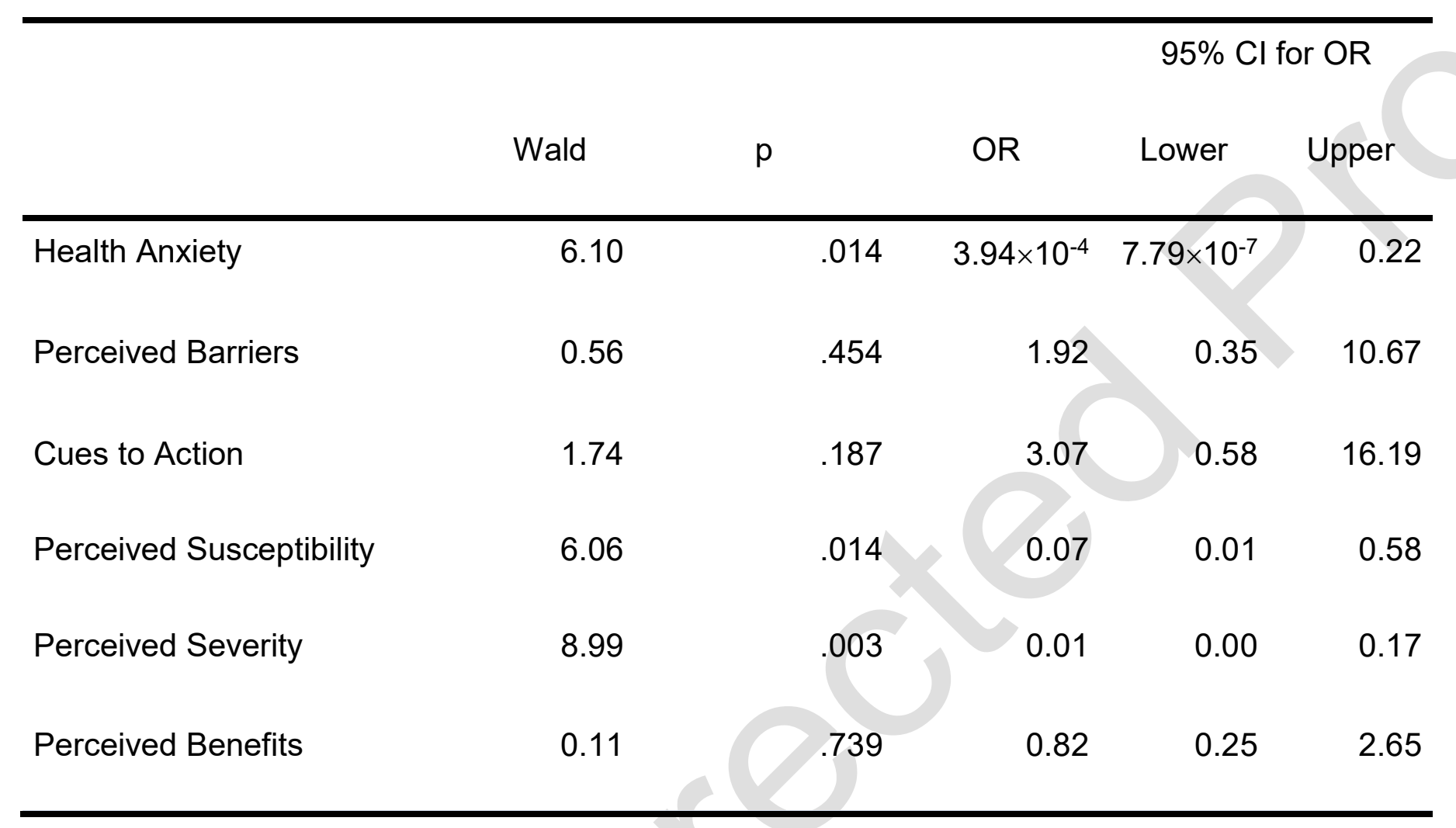


Table 3

Details of Interviewees

\begin{tabular}{llll}
\hline Pseudonym & Age & Sex & Intolerance \\
\hline Alison & 23 & Female & Gluten \\
Aria & 23 & Female & Gluten and Dairy \\
Caleb & 27 & Male & Lactose \\
Emily & 31 & Female & Gluten \\
Hanna & 28 & Female & Gluten \\
Moana & 24 & Female & Lactose \\
\hline
\end{tabular}

